\title{
molecules
}

ISSN 1420-3049

www.mdpi.com/journal/molecules

Article

\section{Inhibitory Effect and Possible Mechanism of Action of Patchouli Alcohol against Influenza A (H2N2) Virus}

\author{
Huaxing Wu ${ }^{1}$, Beili $\mathrm{Li}^{1}$, Xue Wang ${ }^{1}$, Mingyuan Jin ${ }^{1}$ and Guonian Wang ${ }^{2, *}$ \\ 1 Department of Endoscopy, the Third Affiliated Hospital, Harbin Medical University, \\ Harbin 150040, China \\ 2 Department of Anesthesiology, the Third Affiliated Hospital, Harbin Medical University, \\ Harbin 150081, China
}

* Author to whom correspondence should be addressed; E-Mail: gracewhx1964@yahoo.cn; Tel.: +86-451-86298237; Fax: +86-451-86298811.

Received: 13 May 2011; in revised form: 25 July 2011 / Accepted: 28 July 2011 /

Published: 3 August 2011

\begin{abstract}
In the present study, the anti-influenza A (H2N2) virus activity of patchouli alcohol was studied in vitro, in vivo and in silico. The $\mathrm{CC}_{50}$ of patchouli alcohol was above $20 \mu \mathrm{M}$. Patchouli alcohol could inhibit influenza virus with an $\mathrm{IC}_{50}$ of $4.03 \pm 0.23 \mu \mathrm{M}$. MTT assay showed that the inhibition by patchouli alcohol appears strongly after penetration of the virus into the cell. In the influenza mouse model, patchouli alcohol showed obvious protection against the viral infection at a dose of $5 \mathrm{mg} / \mathrm{kg} / \mathrm{day}$. Flexible docking and molecular dynamic simulations indicated that patchouli alcohol was bound to the neuraminidase protein of influenza virus, with an interaction energy of $-40.38 \mathrm{kcal} \mathrm{mol}^{-1}$. The invariant key active-site residues Asp151, Arg152, Glu119, Glu276 and Tyr406 played important roles during the binding process. Based on spatial and energetic criteria, patchouli alcohol interfered with the NA functions. Results presented here suggest that patchouli alcohol possesses anti-influenza A (H2N2) virus properties, and therefore is a potential source of anti-influenza agents for the pharmaceutical industry.
\end{abstract}

Keywords: patchouli alcohol; docking; neuraminidase; MTT; influenza virus; mice 


\section{Introduction}

The influenza virus, which is one of the main causes of acute respiratory infections in humans, can lead to annual epidemics and infrequent pandemics. The two influenza pandemics of the $20^{\text {th }}$ century, “Asian Influenza (1957/H2N2)" and "Hong Kong Influenza (1968/H3N2)" resulted in the deaths of an estimated 2-3 million people globally [1,2]. Today, their descendants continue to cause the majority of influenza infections in humans [3]. So far as it is learned that the most effective antiviral drug is the neuraminidase (NA) inhibitor, which target the NA glycoproteins of influenza A and B virus [4,5].

The release of new virions from the infected cell is a key step in the influenza life cycle and need neuraminidase (NA) to cleave the $\alpha$-ketosidic linkage between terminal sialic acid and an adjacent sugar residue [6]. The NA inhibitors were designed to prevent the key step by blocking the active site of enzyme and thus allow sufficient time for the host immune systems to remove infected viruses [7]. Consistent efforts have been devoted to the development of NA inhibitors, using the crystal structure of the N2 sub-type NA protein [8-15]. Indeed, oseltamivir (Tamiflu) is the representative NA inhibitor that has proven to be uniquely applicable oral drug in clinical practice for the treatment of influenza infection $[4,8,9]$. However, with an increase in medical use, the oseltamivir-resistant strains have been found and probably lead to a large scale outbreak of novel pandemic flu [16,17].

Patchouli alcohol (Figure 1) has been well known for over a century. It is a major constituent of the pungent oil from the East Indian shrub Pogostemon cablin (Blanco) Benth, and widely used in fragrances. Patchouli oil is an important essential oil in the perfume industry, used to give a base and lasting character to a fragrance [16,17]. The essential oil is very appreciated for its characteristic pleasant and long lasting woody, earthy, and camphoraceous odor, as well as for its fixative properties, being suitable for use in soaps and cosmetic products [16,17]. The aerial part of Pogostemon cablin has wildly been used for the treatment of the common cold and as an antifungal agent in China [16,17]. Moreover, the plant is widely used in Traditional Chinese Medicine as it presents various types of pharmacological activity according to the composition of the oil [16,17]. Patchouli alcohol, as the major volatile constituent of patchouli oil, has been found to strongly inhibit H1N1 replication and weakly inhibit B/Ibaraki/2/85 replication [18].

Figure 1. Chemical (A) and stereo (B) structures of patchouli alcohol.

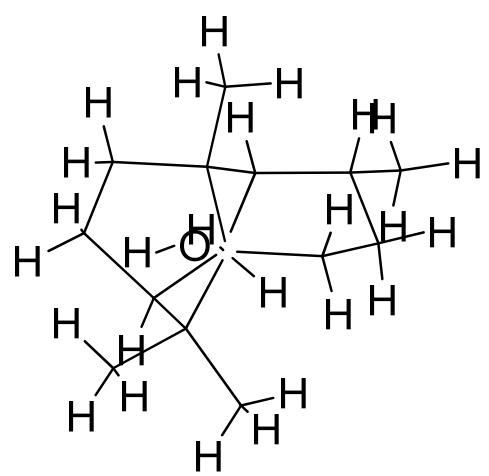

A

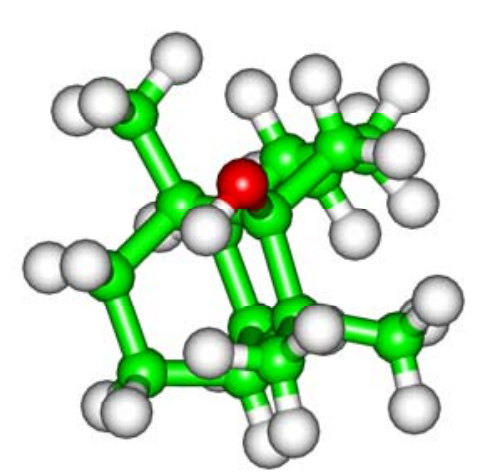

B 
To the best of our knowledge, the anti-influenza virus (H2N2) activities of patchouli alcohol have not been evaluated yet. Therefore, the aim of the present study was to evaluate the anti-influenza A virus (H2N2) activity of patchouli alcohol by MTT assay and mouse influenza model. On such basis, explicitly solvated docking and molecular dynamic (MD) methods were applied to investigative the binding mode involving patchouli alcohol with influenza virus NA protein. We anticipate that the insight into the understanding of inhibiting mechanism will be of value in the rational design of novel anti-influenza drugs.

\section{Results and Discussion}

\subsection{Effect of patchouli alcohol against influenza A (H2N2) virus by MTT assay in vitro}

First the efficacy of patchouli alcohol on influenza A $(\mathrm{H} 2 \mathrm{~N} 2)$ virus replication and cell viability were examined. $\mathrm{CC}_{50}$ was used to express the cytotoxicity of patchouli alcohol on MDCK. The $\mathrm{CC}_{50}$ of patchouli alcohol was above $20 \mathrm{mM}$, which indicated that patchouli alcohol did not affect the growth of MDCK (Table 1). Thus, it seems that the antiviral effects of patchouli alcohol were not due to the cytotoxicity.

Table 1. Anti-influenza A (H2N2) virus activity of patchouli alcohol compared with oseltamivir.

\begin{tabular}{cccc}
\hline \multirow{2}{*}{ Compound } & \multirow{2}{*}{$\mathbf{C C}_{\mathbf{5 0}}{ }^{\boldsymbol{a}}(\boldsymbol{\mu M})$} & \multicolumn{2}{c}{ Influenza A (A/Udorn/307/72) } \\
\cline { 3 - 4 } & $>20.0$ & $\mathbf{I C}_{\mathbf{5 0}}{ }^{\boldsymbol{b}}(\boldsymbol{\mu M})$ & $\mathbf{S I}^{\boldsymbol{c}}$ \\
\hline Patchouli alcohol & $>20.0$ & $0.03 \pm 0.23^{*}$ & $>4.96$ \\
Oseltamivir & $0.031 \pm 0.012$ & $>645.16$ \\
\hline
\end{tabular}

Values in this table represent the mean values $( \pm \mathrm{SD})$ of three independent experiments $(P<0.01)$.

${ }^{a}$ Cytotoxic effect was determined by MTT assay. $\mathrm{CC}_{50}$ was the concentration that showed $50 \%$ cytotoxic effects in MDCK cells; ${ }^{\mathrm{b}}$ Antiviral activity was determined by MTT assay. $\mathrm{IC}_{50}$ was the concentration that inhibited $50 \%$ of Influenza A [(Leningrad/134/17/1957) (H2N2)] virus replication in MDCK; ${ }^{\mathrm{c}}$ The selective index (SI) was calculated as $\mathrm{CC}_{50} / \mathrm{IC}_{50}$. The symbol * indicate a very significant difference $p<0.01$ with respect to positive control (oseltamivir).

Moreover, patchouli alcohol was found to inhibit influenza A $(\mathrm{H} 2 \mathrm{~N} 2)$ virus with an $\mathrm{IC}_{50}$ of $4.03 \pm 0.23 \mu \mathrm{M}$. Based on the $\mathrm{IC}_{50}$ and $\mathrm{CC}_{50}$ values, the selectivity index (SI) was calculated as $>4.96$. It is reported that a SI of 4 or more is appropriate for an antiviral agent [18], suggesting that patchouli alcohol can be judged to have anti-influenza A (H2N2) virus activity.

Until now, it has been found that patchouli alcohol showed dose-dependent anti-influenza virus $(\mathrm{A} / \mathrm{PR} / 8 / 34, \mathrm{H} 1 \mathrm{~N} 1)$ activity, with an $\mathrm{IC}_{50}$ value of $2.635 \mu \mathrm{M}$. Furthermore, it showed weak activity against $\mathrm{B} / \mathrm{Ibaraki} / 2 / 85\left(\mathrm{IC}_{50}=40.82 \mu \mathrm{M}\right)[19]$. With the addition of the above $\mathrm{H} 2 \mathrm{~N} 2$ inhibitory activity, we have a comprehensively view of the anti-influenza activity of patchouli alcohol.

\subsection{Mode of anti-influenza A (H2N2) activity}

Cells were pretreated with patchouli alcohol prior to virus infection (pretreatment cells), viruses were pretreated prior to infection (pretreatment virus), and patchouli alcohol was added during the adsorption period (adsorption) or after penetration of the viruses into cells (replication). Experiments 
were repeated independently three times and data presented are the average of three experiments. The symbols * indicated very significant difference $p<0.01$ with respect to other mode (pretreatment virus, adsorption and pretreatment cell).

As shown in Figure 2, patchouli alcohol showed anti-influenza A (H2N2) virus activity in a timedependent manner. It showed best antiviral activity when added at a concentration of $8 \mu \mathrm{M}$ during the replication period with inhibition of the viral replication of $97.68 \% \pm 2.09 \%$ for influenza A (H2N2) at $72 \mathrm{~h}$. However, no significant effect was detected when patchouli alcohol was used for pretreatment of cells or viruses or when patchouli alcohol was only added during the adsorption phase. These results suggested that the inhibition of influenza A (H2N2) virus by patchouli alcohol appears to occur much more strongly after penetration of the virus into the cell. Besides, biochemical studies have indicated that the bioactivity of NA protein is essential determinant after the replication of influenza A (H2N2) virus [20-22]. Hence, we conclude that the function of NA protein may be suppressed by patchouli alcohol.

Figure 2. Antiviral effect of patchouli alcohol against influenza A (H2N2) virus by incubation at different periods of time during infection.

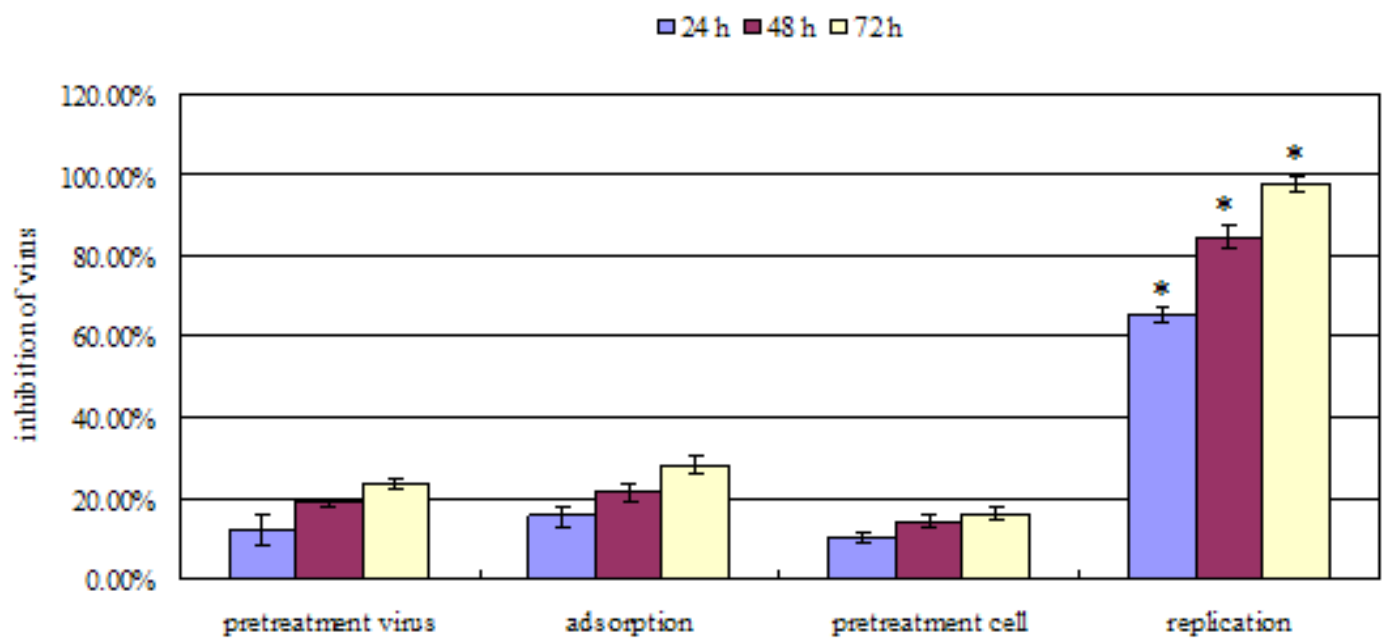

\subsection{Anti-influenza A (H2N2) efficacy in mouse influenza model}

To evaluate the toxicity of patchouli alcohol, the mean value of body weight of mice in each group was statistically analyzed. The mean weights of mice administered at the $2 \mathrm{mg} / \mathrm{kg} / \mathrm{dose}$ oseltamivir, $2 \mathrm{mg} / \mathrm{kg} /$ dose patchouli alcohol and $10 \mathrm{mg} / \mathrm{kg} /$ dose of patchouli alcohol one time daily for 7 days were not significantly different compared with the normal control mice, showing no toxicity of patchouli alcohol and oseltamivir within the testing concentration $(P>0.05)$. Physiological status was observed in virus infection mice. Three days after viral infection, some mice, especially mice in the H2N2 infected control group showed changes in behavior, such as a tendency to huddle, diminished vitality, and ruffled fur, etc. In the mouse influenza model, viral infection leads to loss of body weight and high mortality. Therefore, the efficacy of patchouli alcohol and oseltamivir were evaluated on the basis of survival rate measured for 15 days post-infection, for treated infected animals relative to untreated infected (control) animals. A comparison of efficacy of patchouli alcohol and oseltamivir in vivo mouse influenza model (oral treatment) showed that at a dose of $5 \mathrm{mg} / \mathrm{kg} / \mathrm{day}$, patchouli alcohol 
showed obvious protection against the influenza virus, as the mean day to death was detected as $11.8 \pm 1.1$ (Table 2). When the dose was lowered to $1 \mathrm{mg} / \mathrm{kg} /$ day, patchouli alcohol showed weaker protection (measured by Survivors/total) than that of $5 \mathrm{mg} / \mathrm{kg} / \mathrm{day}$, the mean day to death was $7.5 \pm 1.8$. Whereas oseltamivir at this dose level $(1 \mathrm{mg} / \mathrm{kg} /$ day) showed $50 \%$ protection (measured by survivors/total) against the influenza virus. In the H2N2 infected control group, there were no survivors. In view of both in vitro and in vivo data, we conclude that patchouli alcohol could be used in the treatment of human influenza virus infections.

Table 2. Comparison of efficacy of patchouli alcohol with oseltamivir in the in vivo mouse influenza model (oral treatment).

\begin{tabular}{llll}
\hline Group & $\begin{array}{l}\text { Dose, } \mathbf{m g} / \mathbf{k g} / \mathbf{d a y} \\
\text { (q.d.) }\end{array}$ & Survivors/total & Mean day to death \\
\hline Patchouli alcohol & 5 & $7 / 10^{*}$ & $11.8 \pm 1.1^{*}$ \\
Oseltamivir & 1 & $2 / 10^{*}$ & $7.5 \pm 1.8^{*}$ \\
Control & 1 & $5 / 10^{*}$ & $9.1 \pm 0.3 *$ \\
H2N2 infected control & - & $10 / 10$ & - \\
\hline
\end{tabular}

$* p<0.001$ versus $\mathrm{H} 2 \mathrm{~N} 2$ infected control; ${ }^{a}$ Mean day to death of mice dying prior to day 15 .

\subsection{In silico inhibition mechanism of patchouli alcohol}

Based on the above experiment data, patchouli alcohol is determined to be bound within NA protein. As the total energies and backbone root-mean-square-deviations (RMSD) in Figure 3 indicate, the energy-minimized patchouli alcohol-NA complex has been in equilibrium since about $0.5 \mathrm{~ns}$, and then retains quite stable in the last $19.5 \mathrm{~ns}$. It is consistent with the previous MD results of other NA inhibitors [23-28]. Accordingly, the geometric and energetic analyses were made on the average structures of $0.5 \sim 20.0 \mathrm{~ns}$ MD trajectories, where the system has been already at equilibrium.

Figure 3. The total energies of ensemble (Total energies, A) and time-evolution backbone-atom root mean square deviations (RMSD, B) during the molecular dynamic (MD) simulations for the NA protein complexed with patchouli alcohol.

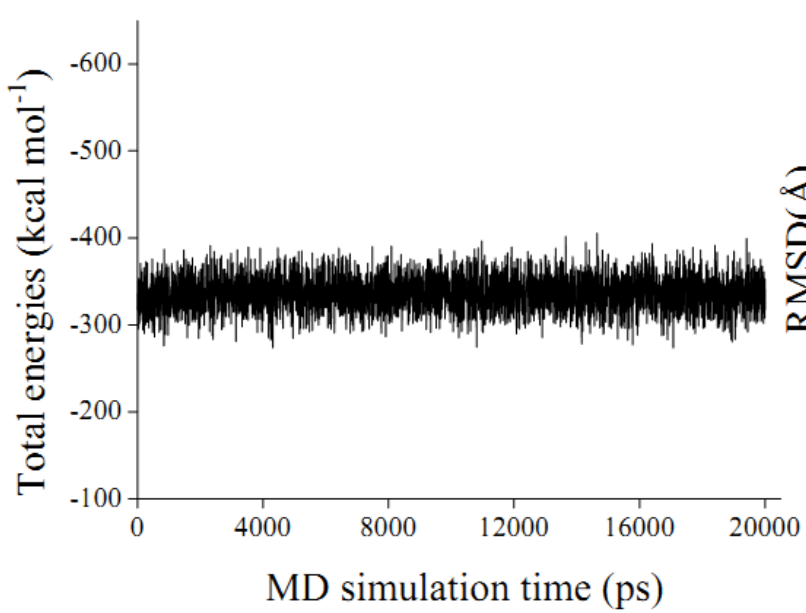

(A)

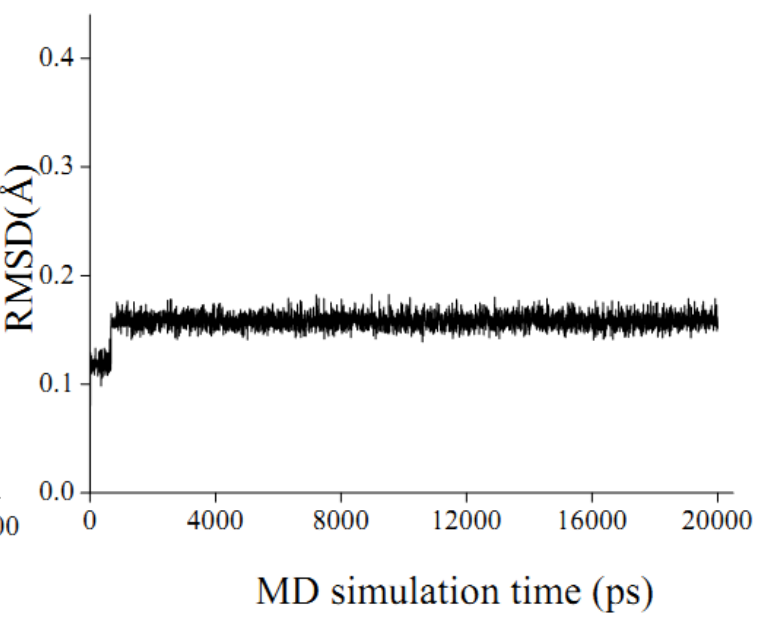

(B) 
The interaction energy $\left(E_{\text {inter }}\right)$ of patchouli alcohol with NA was calculated at $-40.38 \mathrm{kcal} \mathrm{mol}^{-1}$, where the vdW rather than electrostatic interactions were found to play a dominant role, contribute to about $72 \%\left(-29.18 \mathrm{kcal} \mathrm{mol}^{-1}\right)$. As shown in Figure 4, the patchouli alcohol was bound at the active site which also bound to oseltamivir and zanamivir [28]. As Figure 5 shows, the oxygen atom of patchouli alcohol was oriented towards the sidechains of residues Glu119 and Tyr406, with one H-bond formed with each residue. The values of distances in Figure 6 further reveal that the docked complex remains rather stable throughout the simulation, with the average distances of Glu119:OE2 patchouli alcohol:O and Tyr406:OH - patchouli alcohol:O less than $2.8 \AA$. The sum contributions ( $\mathrm{E}_{\text {sum }}$ ) of residues Glu119 and Tyr406 amounted to -8.46 and $-7.37 \mathrm{kcal} \mathrm{mol}^{-1}$, respectively (Table 3). Besides, patchouli alcohol was stabilized by residues Arg118, Asp151, Arg152, Trp178, Ala246, Glu276, Arg292, Asn294 and Gln347, especially residues Asp151, Arg152 and Glu276 (Figure 5 and Table 3). As a matter of fact, residues Asp151, Arg152, Glu119, Glu276 and Tyr406 of the NA protein have already received enough attention from rational drug designs [14,30,31]. The catalytic residues Asp151, Arg152 and Glu276 are crucial to the NA functions and the residues Glu119 and Tyr406 are important to stabilize the NA active sites [32,33]. It suggests that the NA functions will be affected by the presence of patchouli alcohol, consistent with the above experiments. Patchouli alcohol matches with the NA active site and has an acceptable interaction energy. Considering the obvious structure discrepancies against current NA inhibitors, it represents an ideal lead compound for the designs of novel anti-influenza agents.

Figure 4. Patchouli alcohol bound at the NA active site. The Connolly surfaces of the NA active site (in grey) are created using the InsightII 2005 scripts. The patchouli alcohol is represented by ball and stick model. Ribbon colors: Helices, beta sheets, turns and random coils are in red, cyan, green and white, respectively.

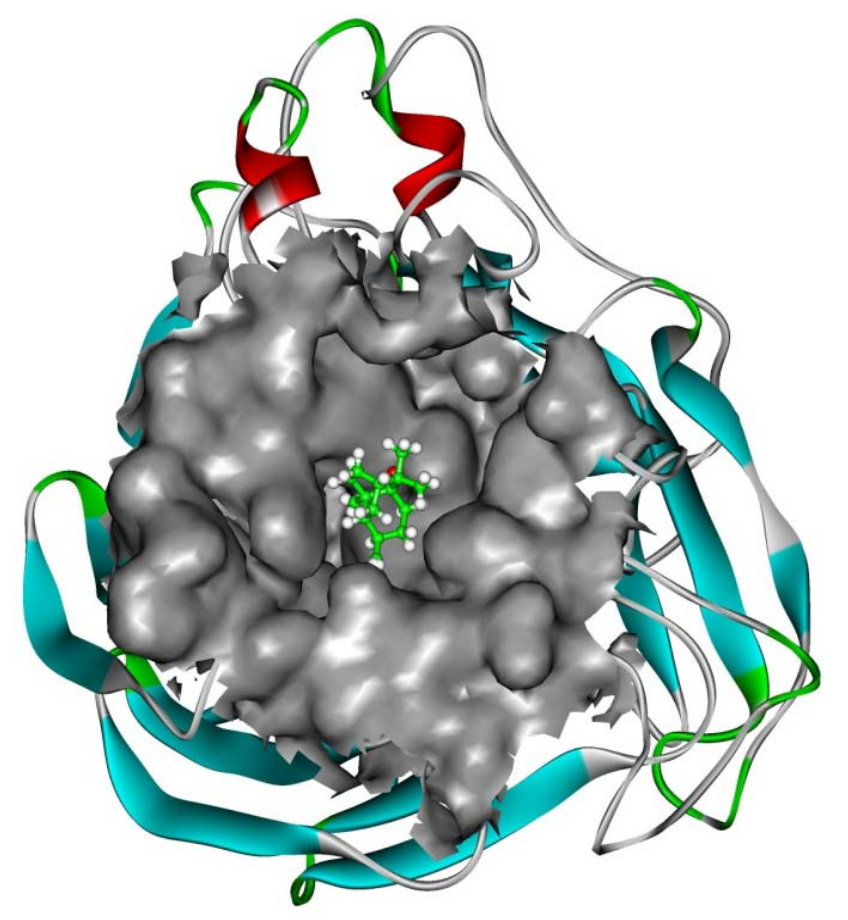


Figure 5. View of the binding mode of patchouli alcohol with the NA active-site residues. Key residues are represented by stick models. Patchouli alcohol is represented by ball and stick model. The hydrogens were avoided for readability. The important H-bonds are labeled in the dashed gold lines.

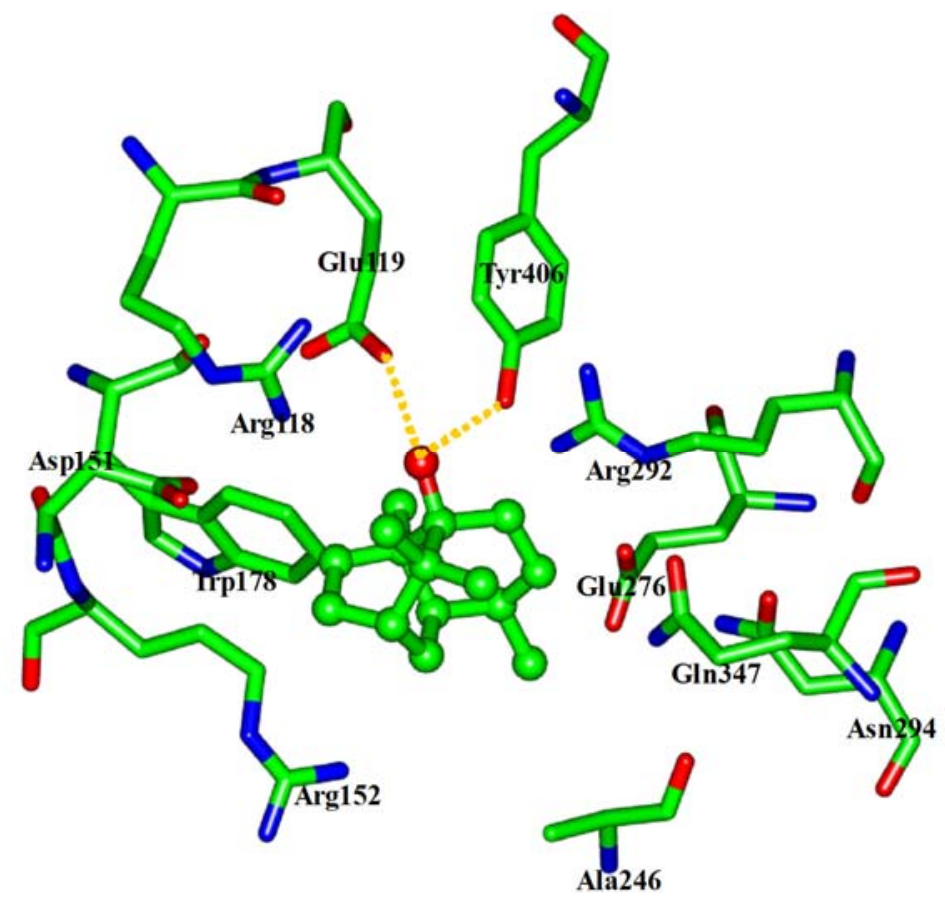

Figure 6. Some time-evolution distances between patchouli alcohol and NA residues.
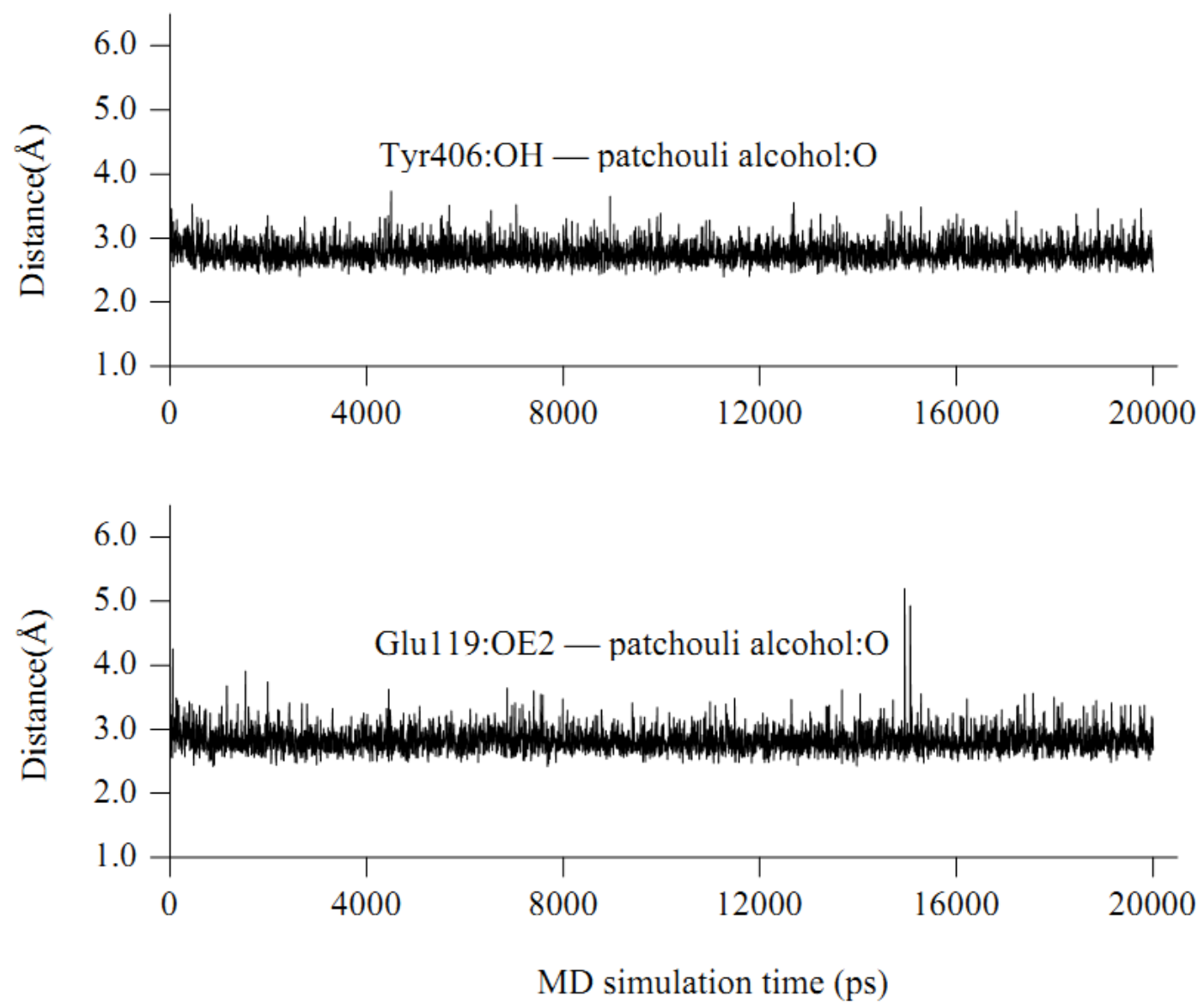
Table 3. The vdW, electrostatic and sum interaction energies $\left(\mathrm{E}_{v d W}, \mathrm{E}_{\text {ele }}\right.$ and $\left.\mathrm{E}_{\text {sum }}\right)$ between patchouli alcohol and the key active-site residues of NA protein ${ }^{a}$.

\begin{tabular}{cccc}
\hline Residue & $\mathbf{E}_{\boldsymbol{v} \boldsymbol{d} \boldsymbol{W}}$ & $\mathbf{E}_{\text {ele }}$ & $\mathbf{E}_{\text {sum }}$ \\
\hline Arg118 & -2.03 & 0.63 & -1.40 \\
Glu119 & -2.18 & -6.28 & -8.46 \\
Asp151 & -2.08 & -0.53 & -2.61 \\
Arg152 & -3.01 & 0.46 & -2.55 \\
Trp178 & -1.83 & 0.01 & -1.82 \\
Ala246 & -1.47 & 0.12 & -1.35 \\
Glu276 & -2.04 & -0.28 & -2.32 \\
Arg292 & -3.51 & 1.10 & -2.41 \\
Asn294 & -0.92 & 0.16 & -0.76 \\
Gln347 & -1.91 & 0.07 & -1.84 \\
Tyr406 & -3.07 & -4.30 & -7.37 \\
\hline
\end{tabular}

${ }^{a}$ Energy units in $\mathrm{kcal} \mathrm{mol}^{-1}$.

\section{Experimental}

\subsection{Materials}

Patchouli alcohol and oseltamivir were obtained from Sigma Chemical Co. (St. Louis, MO, USA, purity $>99 \%$ ) and was stored in glass vials with Teflon sealed caps at $-20 \pm 0.5{ }^{\circ} \mathrm{C}$ in the absence of light.

\subsection{Cell cultures}

MDCK (Madin-Darby canine kidney) was purchased from Harbin Veterinary Research Institute (Harbin, Heilongjiang, China). The cells were grown in monolayer culture with Eagle's minimum essential medium (EMEM) supplemented with 10\% fetal calf serum (FCS), $100 \mathrm{U} / \mathrm{mL}$ penicillin and $100 \mu \mathrm{g} / \mathrm{mL}$ streptomycin. The monolayers were removed from their plastic surfaces and serially passaged whenever they became confluent. Cells were plated out onto 96-well culture plates for cytotoxicity and anti-influenza assays, and propagated at $37{ }^{\circ} \mathrm{C}$ in an atmosphere of $5 \% \mathrm{CO}_{2}$.

\subsection{Viruses}

The influenza strain A/Leningrad/134/17/1957 H2N2) was purchased from National Control Institute of Veterinary Bioproducts and Pharmaceuticals (Beijing, China). Virus was routinely grown on MDCK cells. The stock cultures were prepared from supernatants of infected cells and stored at $-80{ }^{\circ} \mathrm{C}$.

\subsection{Cytotoxicity assay}

The cellular toxicity of patchouli alcohol on MDCK cells was assessed by the MTT method. Briefly, cells were seeded on a microtiter plate in the absence or presence of various concentrations $(20 \mu \mathrm{M}-0.0098 \mu \mathrm{M})$ of patchouli alcohol (eight replicates) and incubated at $37{ }^{\circ} \mathrm{C}$ in a humidified 
atmosphere of $5 \% \mathrm{CO}_{2}$ for $72 \mathrm{~h}$. The supernatants were discarded, washed with PBS twice and MTT reagent $\left(5 \mathrm{mg} / \mathrm{mL}\right.$ in PBS) was added to each well. After incubation at $37{ }^{\circ} \mathrm{C}$ for $4 \mathrm{~h}$, the supernatants were removed, then $200 \mu \mathrm{L}$ DMSO was added and incubated at $37^{\circ} \mathrm{C}$ for another $30 \mathrm{~min}$. After that the plates were read on an ELISA reader (Thermo Molecular Devices Co., Union City, USA) at $570 / 630 \mathrm{~nm}$. The mean OD of the cell control wells was assigned a value of $100 \%$. The maximal non-toxic concentration $\left(\mathrm{TD}_{0}\right)$ and $50 \%$ cytotoxic concentration $\left(\mathrm{CC}_{50}\right)$ were calculated by linear regression analysis of the dose-response curves generated from the data.

\subsection{Anti-influenza A (H2N2) activity in vitro}

Inhibition of virus replication was measured by the MTT method. Serial dilution of the treated virus was adsorbed to the cells for $1 \mathrm{~h}$ at $37{ }^{\circ} \mathrm{C}$. The residual inoculum was discared and infected cells were added with EMEM containing 2\% FCS. Each assay was performed in eight replicates. After incubation for $72 \mathrm{~h}$ at $37^{\circ} \mathrm{C}$, the cultures were measured by MTT method as described above. The concentration of patchouli alcohol and oseltamivir which inhibited virus numbers by $50 \%\left(\mathrm{IC}_{50}\right)$ was determined from dose-response curves.

\subsection{Mode of anti-influenza A (H2N2) activity}

Cells and viruses were incubated with patchouli alcohol at different stages during the viral infection cycle in order to determine the mode of antiviral action. Cells were pretreated with patchouli alcohol before viral infection, viruses were incubated with patchouli alcohol before infection and cells and viruses were incubated together with patchouli alcohol during adsorption or after penetration of the virus into the host cells. Patchouli alcohol was always used at the nontoxic concentration. Cell monolayers were pretreated with patchouli alcohol prior to inoculation with virus by adding patchouli alcohol to the culture medium and incubation for $1 \mathrm{~h}$ at $37^{\circ} \mathrm{C}$. The compound was aspirated and cells were washed immediately before the influenza A (H2N2) inoculum was added. For pretreatment virus, Influenza A (H2N2) was incubated in medium containing patchouli alcohol for $1 \mathrm{~h}$ at room temperature prior to infection of MDCK cells. For analyzing the anti-influenza A (H2N2) inhibition during the adsorption period, the same amount of influenza A (H2N2) was mixed with the drug and added to the cells immediately. After $1 \mathrm{~h}$ of adsorption at $37{ }^{\circ} \mathrm{C}$, the inoculum was removed and DMEM supplemented with $2 \%$ FCS were added to the cells. The effect of patchouli alcohol against influenza A (H2N2) was also tested during the replication period by adding it after adsorption, as typical performed in anti-influenza A (H2N2) susceptibility studies. Each assay was run in eight replicates.

\subsection{Anti-influenza A (H2N2) efficacy in mouse influenza model}

Kunming mice, weighing 18-22 g (6 weeks of age) were purchased from Harbin Veterinary Research Institute Animal Co., Ltd. (Harbin, Heilongjiang, China). First, the toxicity of patchouli alcohol and oseltamivir was assessed in the healthy mice by the loss of body weight compared with the control group ( $2 \%$ DMSO in physiological saline). The mice were orally administered with $10 \mathrm{mg} / \mathrm{kg} / \mathrm{dose}$ patchouli alcohol, $2 \mathrm{mg} / \mathrm{kg} / \mathrm{dose}$ patchouli alcohol or $2 \mathrm{mg} / \mathrm{kg} / \mathrm{dose}$ oseltamivir (dissolved in 2\% DMSO in physiological saline) one time daily for 7 days. The weight of mice was 
determined daily. We conducted procedures according to Principle of Laboratory Animal Care (NIH Publication No. 85 - 23, revised 1985) and the guidelines of the Peking University Animal Research Committee.

Kunming mice were anesthetized with isoflurane and exposed to virus (A/Leningrad/134/17/1957) by intranasal instillation. Drugs were prepared in 2\% DMSO in physiological saline and administered $4 \mathrm{~h}$ prior to virus exposure and continued daily for 5 days. All mice were observed daily for changes in weight and for any deaths. Parameters for evaluation of antiviral activity included weight loss, reduction in mortality and/or increase in mean day to death (MDD) determined through 15 days.

\subsection{Computational methods}

The N2 sub-type neuraminidase crystal structure (PDB code 1IVD) was obtained from the RCSB Protein Data Bank [34]. For convenience, the structure is named as NA hereafter. Geometry and partial atomic charges of the patchouli alcohol (Figure 1) were calculated with the Discover 3.0 module (Insight II 2005) [35] by applying the BFGS algorithm [36] and the consistent-valence force-field (CVFF), with a convergence criterion of $0.01 \mathrm{kcal} \mathrm{mol}^{-1} \AA^{-1}$. The docking and molecular dynamics (MD) simulations were performed by the general protocols in the Insight II 2005 software packages, consistent with the previous literatures [24,26,28,35,37-39]. During the MD simulations, the canonical ensemble (NVT) was employed at normal temperature $(300 \mathrm{~K})$. The MD temperature was controlled by the velocity scaling thermostat [40]. Integrations of the classical equations of motion were achieved using the Verlet algorithm. The systems were solvated in a large sphere of TIP3P water molecules [40] with the radius of $35.0 \AA$, which is enough to hold the ensembles [40]. The MD trajectories were generated using a 1.0-fs time step for a total of $20.0 \mathrm{~ns}$, saved at 5.0-ps intervals. The interaction energies of patchouli alcohol with NA and the respective residues at the NA active site were calculated by the Docking module [35], over the $0.5 \sim 20.0$ ns MD trajectories.

\subsection{Statistical analysis}

All results are expressed as mean values \pm standard deviations (SDs) $(n=3)$. The significance of difference was calculated by one-way analysis of variance, and values $p<0.001$ were considered to be significant.

\section{Conclusions}

In conclusion, patchouli alcohol possesses anti-influenza A (H2N2) virus activity via interference with the NA function that cleaves the $\alpha$-glycosidic bond between sialic acid and glycoconjugate. Our results provide the promising information for the potential use of patchouli alcohol in the treatment of influenza A (H2N2) virus infectious disease. Further mechanistic studies on the anti-influenza A virus activity are needed to support this point of view.

\section{Acknowledgements}

We are grateful for the computing support provided by Harbin Medical University. This research was supported by funds from the Important Research Project of Heilongjiang Province of China (No. 
GB05C402-14), the Outstanding Youth Science Foundation (No. JC200716), and Initial Foundation Project Inside of the Third Affiliated Hospital of Harbin Medical University (No. JJ2005008).

\section{References}

1. Cox, N.J.; Subbarao, K. Global epidemiology of influenza: Past and present. Annu. Rev. Med. 2000, 51, 407-421.

2. Fauci, A.S. Emerging and Re-Emerging Infectious Diseases: Influenza as a Prototype of the Host-Pathogen Balancing Act. Cell 2006, 124, 665-670.

3. Layne, S.P.; Monto, A.S.; Taubenberger, J.K. Pandemic influenza: An inconvenient mutation. Science 2009, 323, 1560-1561.

4. Gubareva, L.V.; Kaiser, L.; Hayden, F.G. Influenza virus neuraminidase inhibitors. Lancet 2000, $355,827-835$.

5. Moscona, A. Neuraminidase inhibitors for influenza. N. Engl. J. Med. 2005, 353, 1363-1373.

6. De Clercq, E.; Neyts, J. Avian influenza A (H5N1) infection: Targets and strategies for chemotherapeutic intervention. Trends Pharmacol. Sci. 2007, 28, 280-285.

7. Garman, E.; Laver, G. Controlling influenza by inhibiting the virus's neuraminidase. Curr. Drug. Targets 2004, 5, 119-136.

8. von Itzstein, M.; Wu, W.Y.; Kok, G.B.; Pegg, M.S.; Dyason, J.C.; Jin, B.; Van Phan, T.; Smythe, M.L.; White, H.F.; Oliver, S.W. Rational design of potent sialidase-based inhibitors of influenza virus replication. Nature 1993, 363, 418-423.

9. Kim, C.U.; Lew, W.; Williams, M.A.; Liu, H.; Zhang, L.; Swaminathan, S.; Bischofberger, N.; Chen, M.S.; Mendel, D.B.; Tai, C.Y. Influenza neuraminidase inhibitors possessing a novel hydrophobic interaction in the enzyme active site: Design, synthesis, and structural analysis of carbocyclic sialic acid analogues with potent anti-influenza activity. J. Am. Chem. Soc. 1997, 119, 681-690.

10. Varghese, J.N.; Smith, P.W.; Sollis, S.L.; Blick, T.J.; Sahasrabudhe, A.; McKimm-Breschkin, J.L.; Colman, P.M. Drug design against a shifting target: A structural basis for resistance to inhibitors in a variant of influenza virus neuraminidase. Structure 1998, 6, 735-746.

11. Atigadda, V.R.; Brouillette, W.J.; Duarte, F.; Babu, Y.S.; Bantia, S.; Chand, P.; Chu, N.; Montgomery, J.A.; Walsh, D.A.; Sudbeck, E.; et al. Hydrophobic benzoic acids as inhibitors of influenza neuraminidase. Bioorg. Med. Chem. 1999, 7, 2487-2497.

12. Gubareva, L.V.; Kaiser, L.; Hayden, F.G. Influenza virus neuraminidase inhibitors. Lancet 2000, $355,827-835$.

13. Brouillette, W.J.; Bajpai, S.N.; Ali, S.M.; Velu, S.E.; Atigadda, V.R.; Lommer, B.S.; Finley, J.B.; Luo, M.; Air, G.M. Pyrrolidinobenzoic acid inhibitors of influenza virus neuraminidase: Modifications of essential pyrrolidinone ring substituents. Bioorg. Med. Chem. 2003, 11, 2739-2749.

14. von Itzstein, M. The war against influenza: Discovery and development of sialidase inhibitors. Nat. Rev. Drug. Discov. 2007, 6, 967-974. 
15. Zhang, J.; Wang, Q.; Fang, H.; Xu, W.; Liu, A.; Du, G. Design, synthesis, inhibitory activity, and SAR studies of hydrophobic p-aminosalicylic acid derivatives as neuraminidase inhibitors. Bioorg. Med. Chem. 2008, 16, 3839-3847.

16. Gubareva, L.V. Molecular mechanisms of influenza virus resistance to neuraminidase inhibitors. Virus Res. 2004, 103, 199-203.

17. Kiso, M.; Mitamura, K.; Sakai-Tagawa, Y.; Shiraishi, K.; Kawakami, C.; Kimura, K.; Hayden, F.G.; Sugaya, N.; Kawaoka, Y. Resistant influenza A viruses in children treated with oseltamivir: Descriptive study. Lancet 2004, 364, 759-765.

18. Kiyohara, H.; Ichino, C.; Kawamura, Y.; Nagai, T.; Sato, N.; Yamada, H. Patchouli alcohol: In vitro direct anti-influenza virus sesquiterpene in Pogostemon cablin Benth. J. Nat. Med. 2011, doi: 10.1007/s11418-011-0550-x.

19. Tsuchiya, Y.; Shimizu, M.; Hiyama, Y.; Itoh, K.; Hashimoto, Y.; Nakayama, M.; Horie, T.; Morita, N. Antiviral activity of natural occurring flavonoids in vitro. Chem. Pharm. Bull. (Tokyo) 1985, 33, 3881-3886.

20. Fan, H.; Ooi, A.; Tan, Y.W.; Wang, S.; Fang, S.; Liu, D.X.; Lescar, J. The nucleocapsid protein of coronavirus infectious bronchitis virus: Crystal structure of its N-terminal domain and multimerization properties. Structure 2005, 13, 1859-1868.

21. Tan, Y.W.; Fang, S.; Fan, H.; Lescar, J.; Liu, D.X. Amino acid residues critical for RNA-binding in the N-terminal domain of the nucleocapsid protein are essential determinants for the infectivity of coronavirus in cultured cells. Nucleic Acids Res. 2006, 34, 4816-4825.

22. Jayaram, H.; Fan, H.; Bowman, B.R.; Ooi, A.; Jayaram, J.; Collisson, E.W.; Lescar, J.; Prasad, B.V. X-ray structures of the N- and C-terminal domains of a coronavirus nucleocapsid protein: implications for nucleocapsid formation. J. Virol. 2006, 80, 6612-6620.

23. Masukawa, K.M.; Kollman, P.A.; Kuntz, I.D. Investigation of Neuraminidase-Substrate Recognition Using Molecular Dynamics and Free Energy Calculations. J. Med. Chem. 2003, 46, 5628-5637.

24. Bonnet, P.; Bryce, R.A. Molecular dynamics and free energy analysis of neuraminidase-ligand interactions. Protein Sci. 2004, 13, 946-957.

25. Bonnet, P.; Bryce, R.A. Scoring binding affinity of multiple ligands using implicit solvent and a single molecular dynamics trajectory: Application to Influenza neuraminidase. J. Mol. Graph. Model. 2005, 24, 147-156.

26. Yang, Z.W.; Yang, G.; Zu, Y.G.; Fu, Y.J.; Zhou, L.J. The conformational analysis and proton transfer of the neuraminidase inhibitors: A theoretical study. Phys. Chem. Chem. Phys. 2009, 11, 10035-10041.

27. Yang, Z.; Yang, G.; Zu, Y.; Fu, Y.; Zhou, L. Computer-Based De Novo Designs of Tripeptides as Novel Neuraminidase Inhibitors. Int. J. Mol. Sci. 2010, 11, 4932-4951.

28. Yang, Z.; Nie, Y.; Yang, G.; Zu, Y.; Fu, Y.; Zhou, L. Synergistic effects in the designs of neuraminidase ligands: Analysis from docking and molecular dynamics studies. J. Theor. Biol. 2010, 267, 363-374.

29. Oakley, A.J.; Barrett, S.; Peat, T.S.; Newman, J.; Streltsov, V.A.; Waddington, L.; Saito, T.; Tashiro, M.; McKimm-Breschkin, J.L. Structural and functional basis of resistance to neuraminidase inhibitors of influenza B viruses. J. Med. Chem. 2010, 53, 6421-6431. 
30. Stoll, V.; Stewart, K.D.; Maring, C.J.; Muchmore, S.; Giranda, V.; Gu, Y.-G.Y.; Wang, G.; Chen, Y.; Sun, M.; Zhao, C.; et al. Influenza neuraminidase inhibitors: Structure-based design of a novel inhibitor series. Biochemistry 2003, 42, 718-727.

31. Aruksakunwong, O.; Malaisree, M.; Decha, P.; Sompornpisut, P.; Parasuk, V.; Pianwanit, S.; Hannongbua, S. On the lower susceptibility of oseltamivir to influenza neuraminidase subtype N1 than those in N2 and N9. Biophys. J. 2007, 92, 798-807.

32. Colman, P.M.; Varghese, J.N.; Laver, W.G. Structure of the catalytic and antigenic sites in influenza virus neuraminidase. Nature 1983, 303, 41-44.

33. Colman, P.M. Influenza virus neuraminidase: Structure, antibodies, and inhibitors. Protein Sci. 1994, 3, 1687-1696.

34. Jedrzejas, M.J.; Singh, S.; Brouillette, W.J.; Laver, W.G.; Air, G.M.; Luo, M. Structures of Aromatic Inhibitors of Influenza Virus Neuraminidase. Biochemistry 1995, 34, 3144-3151.

35. InisghtII Version 2005, 2005 ed.; Accelrys Inc.: San Diego, CA, USA, 2005.

36. Head, J.D.; Zerner, M.C. A Broyden-Fletcher-Goldfarb-Shanno optimization procedure for molecular geometries. Chem. Phys. lett. 1985, 122, 264-270.

37. Yang, Z.W.; Zu, Y.G.; Wu, X.M.; Liu, C.B.; Yang, G. A computational investigation on the interaction mechanisms of neuraminidases and 3-(3-pentyloxy)benzoic acid. Acta Chim. Sin. 2010, 14, 1370-1378.

38. Yang, Z.W.; Wu, N.; Fu, F.J.; Yang, G.; Wang, W.; Zu, Y.G.; Efferth, T. Anti-infectious bronchitis virus (IBV) activity of 1,8-cineole: Effect on nucleocapsid (N) protein. J. Biomol. Struct. Dyn. 2010, 28, 323-330.

39. Affinity User Guide; Accelrys Inc.: San Diego, CA, USA, 2005.

40. Adelman, S.A.; Doll, J.D. Generalized Langevin equation approach for atom/solid-surface scattering: General formulation for classical scattering off harmonic solids. J. Chem. Phys. 1976, 64, 2375-2388.

Sample Availability: Samples of patchouli alcohol are available on request from the authors.

(C) 2011 by the authors; licensee MDPI, Basel, Switzerland. This article is an open access article distributed under the terms and conditions of the Creative Commons Attribution license (http://creativecommons.org/licenses/by/3.0/). 